\title{
Hydrocephalus as a cause of dementia: evaluation by computerised tomography and intracranial pressure monitoring
}

\author{
H.A.CR OCKAR D ${ }^{1}$, K A T H R Y H A N L O N, E. E. D U DA, \\ A N D J.F. M U L L A N
}

From the Section of Neurosurgery and Department of Radiology, University of Chicago Hospitals and Clinics, Chicago, Illinois, USA

SUMMARY To identify those patients with hydrocephalus as a basis for their presenile dementia it is suggested that such patients be screened non-invasively by CT scan. If there is ventricular dilatation and small cortical sulci, intracranial pressure should be monitored for 48 hours to detect ' $B$ ' waves. Patients with significant ' $B$ ' waves $(10 \mathrm{mmHg})$ for at least two hours in the day might benefit from a shunt.

Dementia is becoming an increasing medical and social problem which is compounded by confused diagnostic criteria and uncertain aetiology in many cases. The evaluation of therapy is difficult because of the lack of baseline studies. In young patients, hydrocephalus secondary to trauma, subarachnoid haemorrhage, or meningitis is associated with raised intracranial pressure and dementia. Improvement often follows surgical treatment. However, other patients, usually less than 50-60 years old, who also have hydrocephalus and dementia do not have a permanently raised intracranial pressure so an isolated lumbar puncture pressure measurement may give a false impression. This condition of low pressure or normal pressure hydrocephalus described by Adams et al. (1965) has received wide attention, but the diagnosis and clinical management is still surrounded in controversy (Bannister, 1972; Salmon, 1972; Coblentz et al., 1973; Stein and Langfitt, 1974).

Currently, lumbar pneumo-encephalography is widely used to outline the cerebrospinal fluid (CSF) pathways, and the dynamic situation is evaluated by intrathecal infusion of radioisotopes (RISA scans). Other tests have been devised to measure the rate of formation and absorption of CSF; Coblentz et al. (1973) and Katzman and Hussey (1970). As the tests may not be con-

${ }^{1}$ Present address and address for reprint requests: Department of Neurosurgery, Royal Victoria Hospital, Belfast 12, N. Ireland. Accepted 4 February 1977 clusive, and some are painful and carry a risk to the patient, there are many who consider that invasive testing in such patients is difficult to justify.

The introduction of computerised tomography (CT scan) by Hounsfield (1973) has provided a non-invasive method of detecting ventricular enlargement, widening of sulci, or less obvious findings associated with early onset dementia, such as multiple focal cerebral infarctions, or small unsuspected tumours or cysts. Huckman et al. (1975) have described normal measurements of the lateral ventricles and cortical sulci on CT scans, estimated the degree of cortical atrophy, and correlated them with the neurological condition. They also described a group of demented patients with ventricular enlargement but normal cortical sulci. If ventricular enlargement is due to CSF pathway obstruction, even of an intermittent type, a shunting procedure, such as a ventriculoatrial shunt, might help the dementia. In such patients, Symon and Dorsch (1975) and Chawla et al. (1974) have demonstrated periods of raised intracranial pressure with characteristic pressure wave patterns. The identification of these pressure changes is probably more useful than the more sophisticated study of the exact cause of the CSF production/absorption imbalance.

This preliminary report attempts to correlate the CT scan measurements with the findings of intracranial pressure monitoring and to determine if these two safe tests are sufficient for the detection of normal pressure hydrocephalus. 


\section{Measurements and methods}

Nineteen adult patients have been studied, 11 men and eight women. Twelve presented with varying degrees of dementia, ranging from a deterioration in mental ability to gross confusional states associated with difficulty in walking, increased tone in the lower limbs, and incontinence. The other seven patients had been treated previously for communicating hydrocephalus and were admitted to hospital because their CSF shunts were not functioning satisfactorily. In four, the original shunting procedure had been carried out for communicating hydrocephalus after meningitis or subarachnoid haemorrhage; two had congenital lesions and in one the original shunt was inserted for possible normal pressure hydrocephalus (NPH).

Intracranial pressure was measured for at least two days (range two to five days) using a ventricular catheter inserted through a frontal burr hole and connected to an external transducer (Statham p2 $3 \mathrm{~V}$ ). The decision to insert a shunt or to revise the existing system was entirely on clinical grounds and intracranial pressure measurements. After some preliminary studies, some patterns and levels of intracranial pressure were arbitrarily taken as evidence of obstruction. These were: (a) a raised, basal, or resting intracranial pressure (above $10 \mathrm{mmHg}$ ) measured when the patient was alert and lying still; (b) the presence of Lundberg 'A' and 'B' waves (Lundberg, 1960) which were at least $10 \mathrm{mmHg}$ higher than resting intracranial pressure. These pressure wave patterns are generally accepted to indicate abnormal intracranial dynamics, and, in the case of hydrocephalus, CSF pathway obstruction. 'A' waves are transient elevations of intracranial pressure lasting five to 20 minutes; ' $B$ ' waves have a regular 'saw-tooth' pattern with a periodicity of 30 seconds to two minutes (Fig 1); (c) the wave patterns must have been present for at least $10 \%$ of the time monitored-that is, at least two hours in every day, whether the patient was awake or asleep.

The following measurements from the preoperative CT scans (Huckman et al., 1975) were performed (by EED) without knowledge of the intended surgical procedure. These were (in $\mathrm{mm}$ ): (a) the distance between the most lateral parts of the frontal horns; (b) the width of the lateral ventricles in the thalamic region; (c) the sum of (a) and (b) taken as the measurement of ventricular size; and (d) the total width of the four largest cortical sulci seen in the highest three tomographic cuts.

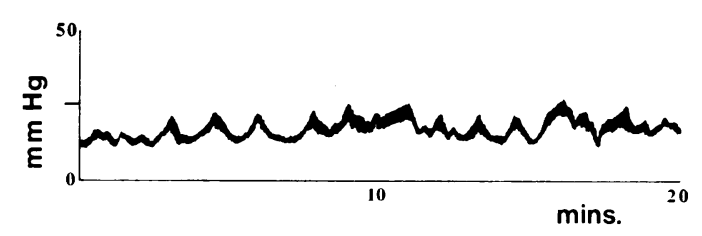

PRE-SHUNT

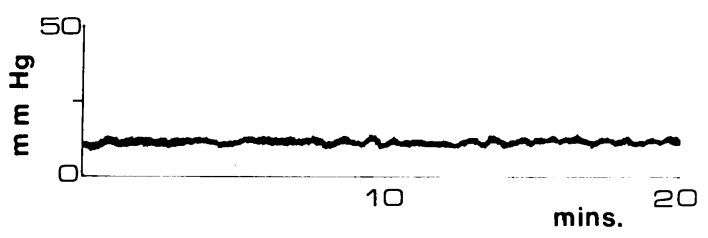

POST-SHUNT

Fig. 1 ICP record of a patient with NPH. Note the saw-tooth pattern of ' $B$ ' waves which were abolished by insertion of ventriculo-atrial shunt.

An example of cortical atrophy with large ventricles and prominent sulci is shown in Fig. 2. The CT scan associated with normal pressure hydrocephalus is shown in Fig. 3.

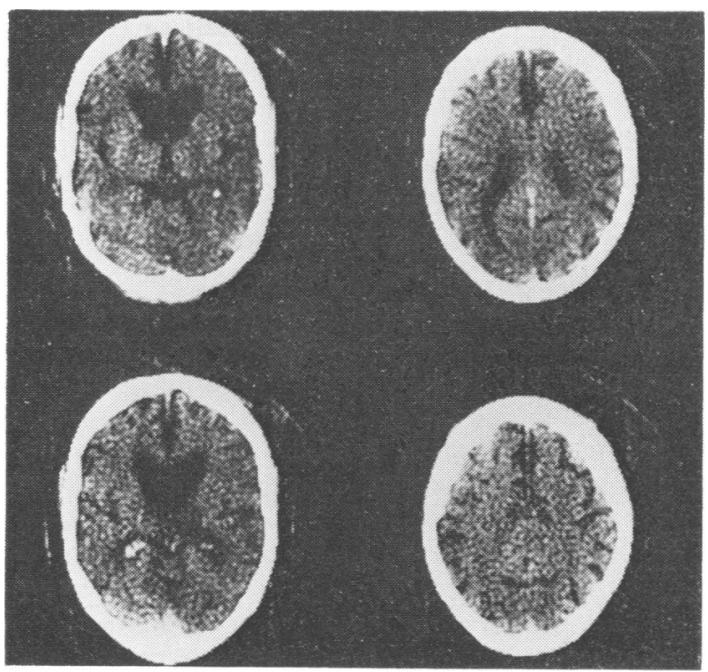

Fig. 2 CT scan of a patient with ventricular enlargement and prominent cortical sulci. There were no ' $B$ ' waves in his ICP record, and he was considered to have cerebral atrophy.

\section{Results}

Of the 12 patients presenting with dementia, eight were judged to have normal pressure hydrocephalus, one had a persistently raised resting 


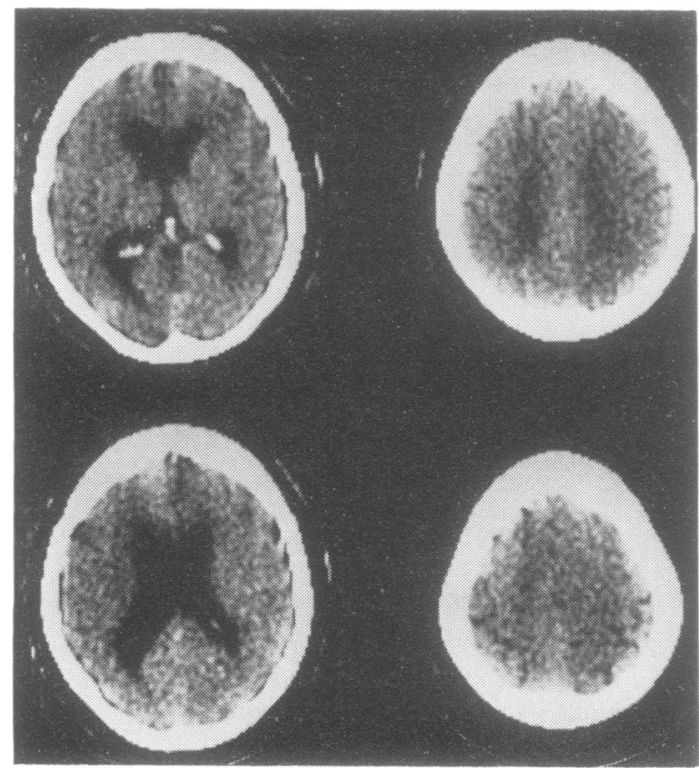

Fig. 3 This patient had numerous ' $B$ ' waves, and $C T$ scan showed dilated lateral ventricles and small sulci. This was a case of NPH.

intracranial pressure and, therefore, was a case of high pressure hydrocephalus. The remaining three had no pressure waves and because of the finding of large sulci on CT scan were considered to have cerebral atrophy.

In the group (Table 1) judged to have normal pressure hydrocephalus the resting intracranial pressure (mean $+\mathrm{SD}$ ) was $11.7 \pm 6.7 \mathrm{mmHg}$ (range 6-20 $\mathrm{mmHg}$ ), and the ' $B$ ' waves had a mean height of $17.1 \pm 8.9 \mathrm{mmHg}$ (range $10-30 \mathrm{mmHg}$ ) above the resting pressure. ' $B$ ' waves occupied between $17-91 \%$ of the monitoring times. There were no plateau or ' $A$ ' waves in this group. After the insertion of a shunt the resting intracranial pressure was unchanged but the ' $B$ ' waves

Table 1 Summary of the mean (and Standard Deviation) ICP results for the three groups. The NPH group is similar to the group presenting with nonfunctioning shunts, and both of these are quite different from the three with large cortical sulci on CT scan considered to have cerebral atrophy (see text). Three other patients are not included in this table-one with high pressure and two with shunts judged to be functioning satisfactorily.

\begin{tabular}{llclr}
\hline & Number $\begin{array}{l}\text { Resting ICP } \\
m m H g\end{array}$ & $\begin{array}{l}\text { ICP waves } \\
m m H g\end{array}$ & $\begin{array}{l}\% \text { Time } \\
\text { abnormal }\end{array}$ \\
\hline NPH & 8 & $11.7(6.7)$ & $17.1(8.9)$ & $41.5(28.1)$ \\
Shunt revision & 5 & $17.4(11.1)$ & $22.8(12.4)$ & $63.0(35.0)$ \\
Cerebral atrophy & 3 & $8.3(2.5)$ & $5.3(4.0)$ & $1.7(2.9)$ \\
\hline
\end{tabular}

disappeared, although in several cases there was a ripple variation in intracranial pressure (Fig. 1). The pressure characteristics of this group were remarkably similar to those of the five patients previously treated for high pressure hydrocephalus who required shunt revision. The resting intracranial pressure and height of waves were similar but ' $B$ ' wave activity was more frequently seen in those requiring a shunt revision, and, in one case there were prominent ' $A$ ' waves.

The three patients considered to have cortical atrophy had very different records of intracranial pressure. The resting pressure was similar but there were hardly any ' $B$ ' waves $(0-5 \%$ of the time monitored), and they were not considered to be significant.

The CT scan measurements in those with normal pressure hydrocephalus were compared with those who had normal intracranial pressure records (Table 2). There was no difference in ventricular measurements between the two groups (measurements A, B, and C) but a significant difference in the estimates of sulci (measurement D), suggesting that the decreased brain volume resulted from atrophy.

Table 2 Mean and SD of CT scan measurements after Huckmann et al. (1975) of the NPH group who were shunted and those with cerebral atrophy. Only the measurements of cortical sulci $(D)$ were significantly different.

\begin{tabular}{lllll}
\hline & \multicolumn{4}{l}{ CT scan measurements $($ in $\mathrm{mm})$} \\
\cline { 2 - 5 } & $A$ & $B$ & $C$ & $D$ \\
\hline Shunted & $11.5(2.2)$ & $7.1(1.6)$ & $18.6(3.4)$ & $3.1(0.3)$ \\
Not shunted & $14.0(3.0)$ & $8.5(2.8)$ & $22.5(5.6)$ & $6.0(0.0)$ \\
\hline
\end{tabular}

\section{Discussion}

The patterns of the intracranial pressure of the five patients who had shunt revisions and the eight considered to have normal pressure hydrocephalus were remarkably similar in resting pressure and the presence of significant ' $B$ ' waves. These findings are similar to those reported by Symon and Dorsch (1975) and are taken as evidence that at least some patients with dementia have periods of raised intracranial pressure. Rubin et al. (1976) have shown that in chronic experimental hydrocephalus in cats, there is axonal disruption in the periventricular white matter. Granholm (1976) has shown by computerised tomography that in hydrocephalus there is periventricular oedema which disappears with shunting. If these findings can be transposed to the situation of normal pressure hydrocephalus, 
it is likely that intermittent rises of intracranial pressure left unchecked would produce damage to the periventricular white matter and limbic system. If the pressure can be controlled at an early stage in the disease the damage might be minimal, and, if due to oedema, reversible.

While we and other workers have shown that some demented patients have raised intracranial pressure, it is not yet clear whether reducing the pressure improves their condition. There are some reasons for this uncertainty. Firstly, the methods of diagnosis have been uncertain. In a review of their cases, Stein and Langfitt (1974) found that the various tests, which included isotope studies and measurement of cerebral blood flow, were not conclusive in their ability to predict the outcome of a shunting procedure. Only in those with a definite history of previous meningitis, trauma and so on, was there an improvement after insertion of a shunt; without such a history only $23 \%$ improved.

It is our contention that patients should be screened non-invasively by CT scan. The presence of large cortical sulci with dilated ventricles is taken as evidence of cerebral atrophy and such patients should be spared further needless investigation: Enlarged ventricles alone might suggest a degree of CSF pathway obstruction, and in a suitable patient the procedure most likely to evaluate this would be monitoring of intracranial pressure. A high resting pressure and the presence of significant ' $B$ ' waves for at least $10 \%$ of the time monitored are taken as evidence of CSF obstruction.

It will be rare that everything will be so defined. It is possible that some demented patients have a combination of cerebral atrophy from a degenerative disease and raised intracranial pressure because of CSF pathway obstruction. The results of shunting these patients might be disappointing. Also, patients with dementia (Kay, 1962), or ventricular enlargement (Lönnum and Kornstad, 1974), have reduced expectancy of life, so it will be difficult to perform long-term assessments. It is, therefore, an important requirement in the study of dementia to use widely accepted and objective psychometric measurements, to determine the role of raised intracranial pressure in this condition. A trial in several centres is being planned.

\section{Conclusion}

Twelve patients with dementia and five patients who had already been treated for hydrocephalus and required a shunt revision were studied by computerised tomography and then by measurements of intracranial pressure for at least 48 hours. Eight patients with dementia had similar pressure patterns to those who required a shunt revision. They also had enlarged ventricles on CT scan and were considered to have normal pressure hydrocephalus. Three demented patients had no rise in intracranial pressure and no ' $B$ ' waves; on $C T$ scan they had large sulci. We suggest that the use of CT scanning to detect ventricular dilatation and enlarged sulci would identify patients with definite cortical atrophy who have nothing to gain from a shunting operation. In those patients with ventricular enlargement alone, $10 \mathrm{mmHg}$ ' $B$ ' waves for more than $10 \%$ of the time monitored would be evidence of CSF pathway obstruction. Such patients might benefit from a shunt.

\section{References}

Adams, R. D., Fisher, C. M., Hakim, S., Ojemann, R. G., and Sweet, W. H. (1965). Symptomatic occult hydrocephalus with 'normal' cerebrospinal fluid pressure. New England Journal of Medicine, 273, 117-126.

Bannister, C. M. (1972). A report of eight patients with low pressure hydrocephalus treated by CSF diversion with disappointing results. Acta Neurochirurgica (Wein), 27, 11-15.

Chawla, J. C., Hulme, A., and Cooper, R. (1974). Intracranial pressure in patients with dementia and communicating hydrocephalus. Journal of Neurosurgery, 40, 376-380.

Coblentz, R. M., Mattis, S., and Zingesser, L. H. (1973). Presenile dementia. Archives of Neurology (Chicago), 29, 299-308.

Granholm, L. (1976). An explanation of the reversible memory defect in hydrocephalus: In Intracranial Pressure. Vol. 3, pp. 173-176. Edited by J. W. J. Becks, D. A. Bosch, and M. Brock. Springer Verlag: Berlin.

Hounsfield, G. N. (1973). Computerised transverse axial scanning Part I. Description of system. British Journal of Radiology, 46, 1016-1022.

Huckman, M. S., Fox, J., and Topel, J. (1975). The validity of criteria for evaluation of cerebral atrophy by computerised tomography. Radiology, 116, 85-92.

Katzman, R., and Hussey, F. (1970). A simple constant infusion manometric test for measurement of cerebrospinal fluid absorption. I. Rationale and method. Neurology (Minneapolis), 20, 534-544.

Kay, D. W. K. (1962). Outcome and cause of death in mental disorders of old age: A long term follow up of functional and organic psychoses. Acta Psychiatrica et Neurologica Scandinavica, 38, 249276.

Lönnum, A., and Kornstad, S. (1974). Long term prognosis of patients with central cerebral ventricular enlargement. Acta Neurologica Scandinavica, 50, 409-433. 
Lundberg, N. G. (1960). Continuous recording and control of ventricular fluid pressure in neurosurgical patients. Acta Psychiatrica Scandinavica, 36 (Suppl. 149).

Rubin, R. C., Hochwald, G. M., and Tiell, M. (1976). Hydrocephalus: I. Histological and ultrastructural changes in the pre-shunting cortical mantle. Surgical Neurology, 5, 109-114.

Salmon, J. H. (1972). Adult hydrocephalus: evaluation of shunt therapy in 80 patients. Journal of
Neurosurgery, 37, 423-428.

Stein, S. C., and Langfitt, T. W. (1974). Normal pressure hydrocephalus: predicting the results of cerebrospinal fluid shunting. Journal of Neurosurgery, 41, 463-470.

Symon, L., and Dorsch, N. W. C. (1975). Use of long term intracranial pressure measurement to assess hydrocephalic patients prior to shunt surgery. Journal of Neurosurgery, 42, 258-273. 